\title{
New Concepts of Homogenization Applied in Rough Surface Hydrodynamic Lubrication
}

\author{
Andreas Almqvist $^{a, *}$, Dag Lukkassen ${ }^{b}$, Annette Meidell $^{b}$ \\ and Peter Wall ${ }^{c}$ \\ ${ }^{a}$ Division of Machine Elements, Luleå \\ University of Technology, SE-971 87 Luleå, Sweden. \\ ${ }^{b}$ Narvik University College, N-8505 Narvik, Norway. \\ ${ }^{c}$ Department of Mathematics, Luleå \\ University of Technology, SE-971 87 Luleå, Sweden.
}

\begin{abstract}
This work introduces a new concept of homogenization that enables efficient analysis of the effects of surface roughness representations obtained by measurements in applications modeled by the Reynolds equation. Examples of such applications are trust- and journal- bearings. The numerical analysis of these types of applications requires an extremely dense computational mesh in order to resolve the surface roughness, suggesting some type of averaging. One such method is homogenization, which has been applied to Reynolds type equations with success recently. This approach is similar to the technique proposed by Patir and Cheng, who introduced flow factors determining the hydrodynamic action due to surface roughness. The difference is, however, that the present technique has a rigorous mathematical support. Moreover, the recipe to compute the averaged coefficients is simple without any ambiguities. Using either the technique proposed by Patir and Cheng or homogenization, the coefficients determining the averaged Reynolds equation are obtained by solving differential equations on a local scale. Unfortunately, this is detrimental when investigating the effects induced by real, measured, surface roughness, even though these local problems may be solved in parallel.

The present work presents a solution by applying the technique based on bounds. This technique transforms the stationary Reynolds equation into two computationally feasible forms, one for the upper bound and one for the lower bound, where the flow factors are obtained by straightforward integration. Together with the preciseness of these bounds, the bounds approach becomes an eminent tool suitable for investigating the effect of real, measured, surface roughness on hydrodynamic performance.
\end{abstract}

Keywords: Surface roughness / topography; Reynolds equation; Flow factors; Homogenization; Lubrication;

Bounds

${ }^{*}$ Corresponding author. Tel.: +46920492407; fax: +46920491047. E-mail address: Andreas.Almqvist@ltu.se (A. Almqvist) 


\section{Nomenclature}

$\Lambda \quad$ Constant, $\Lambda=6 \mu U+$ Pa m

$\mu \quad$ Dynamic viscosity at ambient pressure $\quad$ Pa s

$\nabla \quad$ Operator, $\nabla=\left(\partial / \partial x_{1}, \partial / \partial x_{2}\right)$

$\nabla_{y} \quad$ Operator, $\nabla_{y}=\left(\partial / \partial y_{1}, \partial / \partial y_{2}\right)$

$\Omega \quad$ Open bounded subset of $\mathbb{R}^{2}$

$\varepsilon \quad$ Parameter describing the local length scale $(\varepsilon>0)$

$A_{0} \quad$ Homogenized matrix

$B_{0} \quad$ Homogenized vector

$h_{0} \quad$ Global film thickness $\quad \mathrm{m}$

$h_{1} \quad$ Roughness part, positioned on the stationary surface m

$h_{\varepsilon} \quad$ Film thickness $\mathrm{m}$

$h_{\text {min }} \quad$ Global film thickness minimum, $h_{\min }=\min \left(h_{0}\right) \quad \mathrm{m}$

$L_{i} \quad$ Length of solution domain in the $x_{i}$-direction $\quad \mathrm{m}$

$p^{+} \quad$ Pressure, upper arithmetic-harmonic mean bound $\quad \mathrm{Pa}$

$p^{-} \quad$ Pressure, lower arithmetic-harmonic mean bound $\quad \mathrm{Pa}$

$p_{0} \quad$ Pressure, homogenized solution $\quad \mathrm{Pa}$

$p_{\varepsilon} \quad$ Pressure, deterministic solution $\quad P a$

$p_{R V}^{+} \quad$ Pressure, upper Reuss-Voigt type bound $\quad \mathrm{Pa}$

$p_{R V}^{-} \quad$ Pressure, lower Reuss-Voigt type bound $\quad \mathrm{Pa}$

$U \quad$ Velocity of the moving surface $\mathrm{m} \mathrm{s}^{-1}$

$x \quad$ Spatial coordinate, $x=\left(x_{1}, x_{2}\right)$

$Y \quad$ Y-cell, $Y=[0,1]^{2}$

$y \quad$ Local spatial coordinate, $y=\left(y_{1}, y_{2}\right)$ 


\section{Introduction}

To increase the hydrodynamic performance in different machine elements during lubrication, e.g. journal bearings and thrust bearings, it is important to understand the influence of surface roughness. In this paper the equation governing the hydrodynamic action is the incompressible, stationary Reynolds equation. The objective is to derive a method that enables efficient analysis of the influence of surface roughness on hydrodynamic performance. The literature offers many different methods with a similar objective. Among them, the flow factor based approach presented by Patir and Cheng [1] is maybe the most well-known. It has certainly made an important impact, been frequently used and further developed since then.

This paper considers another method called homogenization, which is general and concerns the study of partial differential equations with rapidly oscillating coefficients, see Jikov et al. [2]. One branch considers various delicate convergence problems that lead to rigorously defined averaged properties. For application of homogenization to the type of Reynolds equation studied in this work, see e.g Almqvist and Dasht [3], Elrod [4], Bayada and Faure [5], Kane and Bou-Saï [6] and Wall [7]. Examples of other problems related to Reynold type equations and analyzed by homogenization are $[8,9,10,11,12,13,14,15,16]$.

Similar to the flow factor based approach, the application of homogenization imposes no restrictions other than the assumptions introduced when deriving the Reynolds equation and that the surface roughness representation is periodically repeated. As well, the problem can be divided into two different parts, one for the local scale describing surface roughness and one for the global scale describing the geometry of the application. The homogenization process differs from the flow factor based approach in its determination of global problem coefficients or flow factors, that are unambiguously obtained through the solution of rigorously well defined local problems. An other important aspect is repeatability, which is a major disadvantage of the flow factor based approaches. The homogenization process is however perfectly repeatable. Unfortunately, applying any available approach will become a difficult task when real, measured rough surfaces are considered. This is since they rely on the possibility of solving a set of differential equations at the surface roughness scale, which leads to enormously time-consuming, if not impossible, analysis of real surfaces.

Another branch within homogenization to consider is the problem of finding upper and lower bounds of the averaged properties. The ideas of finding bounds have proved to be useful in different physical applications, such as composite engineering see e.g. [17, 18] and the references given there. Recent mathematical work [19] presents a type of bounds that may be used to analyze the stationary Reynolds equation. This paper demonstrates that these bounds may be used for efficient analyzes of the influence of surface roughness on hydrodynamic performance. Moreover, by using the ideas presented in [19], a less precise type of bounds is derived here. The benefit of these less precise bounds is that they lead to even more computationally beneficial forms. Nevertheless, it is shown here that in many situations they can be used to obtain sufficiently accurate predictions. This makes the bounds 
approach eminent when applied to investigations regarding the effects induced by real, measured rough surfaces on the hydrodynamic performance. To our knowledge, this is the first time the usefulness of bounds is clearly demonstrated for these types of applications.

\section{The governing Reynolds equation}

In this work, the film thickness is divided into two parts representing the global scale film thickness and the local scale surface roughness. For this purpose an auxiliary function

$$
h(x, y)=h_{0}(x)+h_{1}(y),
$$

where $h_{1}$ is to be considered as a $Y$-periodic function.

Assuming a smooth moving surface and a rough stationary surface the film thickness may be modeled by

$$
h_{\varepsilon}(x)=h_{0}(x)+h_{1}(x / \varepsilon), \quad \varepsilon>0,
$$

where $\varepsilon$ is a parameter introduced for variation of surface roughness wavelength. As an example, a schematic illustration of a slider bearing defined by $h_{\varepsilon}$ is given in Fig. 1.

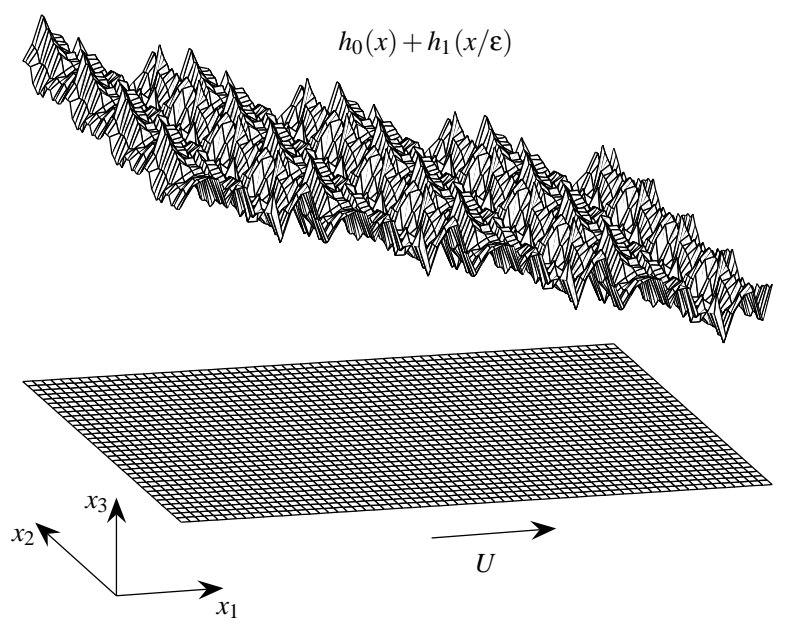

Figure 1: A schematic slider bearing description.

Let $\mu$ be the viscosity of the lubricant and assume that the only smooth surface is moving with the speed $U$ and that the motion only takes place in the $x_{1}$ direction. The governing Reynolds equation is then given by

$$
\nabla \cdot\left(h_{\varepsilon}^{3}(x) \nabla p_{\varepsilon}(x)\right)=\Lambda \frac{\partial h_{\varepsilon}(x)}{\partial x_{1}} \quad \text { on } \Omega \subset \mathbb{R}^{2},
$$

where $p_{\varepsilon}$ is the pressure, assuming $p_{\varepsilon}=0$ at the boundaries and $\Lambda=6 \mu U$. This constitutes an incompressible and iso-viscous model of stationary fluid flow. Alternatively, the pressure $p_{\varepsilon}$ can be found by solving the variational 
problem corresponding to (11), i.e.

$$
\min _{p} \int_{\Omega}\left(\frac{1}{2} h_{\varepsilon}^{3} \nabla p \cdot \nabla p-\Lambda h_{\varepsilon} e_{1} \cdot \nabla p\right) d x
$$

For small values of $\varepsilon$, the function $h_{\varepsilon}$ oscillates rapidly. This implies that finding $p_{\varepsilon}$ by a direct numerical treatment of the deterministic problems (11) and (2) is very time consuming due to the very dense discretization mesh needed to resolve the surface roughness. For sufficiently small values of $\varepsilon$ it is even impossible. In these situations the concept of homogenization constitutes a valuable tool, rigorously averaging the effect of the surface roughness induced variation in the coefficients of the governing equation.

\section{Homogenization}

In this section some preliminary results and consequences of the homogenization theory are given. For further information, we refer to [2]. Note that without loss of generality, the assumption $Y=[0,1]^{2}$ is applied in the subsequent analysis. Let the "determinsitic energy" $F_{\varepsilon}\left(\nabla p_{\varepsilon}\right)$ be defined as

$$
F_{\varepsilon}\left(\nabla p_{\varepsilon}\right)=\min _{p} \int_{\Omega} \frac{1}{2} h_{\varepsilon}^{3} \nabla p \cdot \nabla p-\Lambda h_{\varepsilon} e_{1} \cdot \nabla p d x
$$

One well-known result that may be proved by using $\Gamma$-convergence is the following convergence of "energies"

$$
F_{\varepsilon}\left(\nabla p_{\varepsilon}\right) \rightarrow F_{0}\left(\nabla p_{0}\right)=\min _{p} F_{0}(\nabla p) \quad \text { as } \varepsilon \rightarrow 0
$$

Here

$$
F_{0}(\nabla p)=\int_{\Omega} f_{0}(x, \nabla p) d x
$$

where the homogenized energy density function $f_{0}$ is given in terms of a local minimization problem

$$
f_{0}(x, \xi)=\min _{w \in W_{p e r}} \int_{Y} \frac{1}{2} h^{3}(x, y)\left(\xi+\nabla_{y} w\right) \cdot\left(\xi+\nabla_{y} w\right)-\Lambda h(x, y) e_{1} \cdot\left(\xi+\nabla_{y} w\right) d y,
$$

where $W_{p e r}$ is the set of $Y$-periodic functions and $e_{1}=(1,0)$. Also, observe that the integral is evaluated only over one cell of periodicity. To be able to find $F_{0}\left(\nabla p_{0}\right)$ it is necessary to find $f_{0}(x, \xi)$, though this is a delicate problem. Let us therefore discuss how this may be done. We observe that

$$
f_{0}(x, \xi)=\min _{w \in W_{p e r}} \int_{Y} \frac{1}{2} h^{3} \nabla_{y} w \cdot \nabla_{y} w+\left(h^{3} \xi-\Lambda h e_{1}\right) \cdot \nabla_{y} w d y+\int_{Y} \frac{1}{2} h^{3} \xi \cdot \xi-\Lambda h e_{1} \cdot \xi d y,
$$

where the second integral does not depend on $w$. That is, if $w_{\xi}$ is a minimizer in (3), then $w_{\xi}$ is also a minimizer in

$$
\min _{w \in W_{p e r}} \int_{Y} \frac{1}{2} h^{3} \nabla_{y} w \cdot \nabla_{y} w-\left(\Lambda h e_{1}-h^{3} \xi\right) \cdot \nabla_{y} w d y .
$$

This implies that the minimizer $w_{\xi}$ also solves the Euler equation corresponding to (4). That is

$$
\int_{Y} h^{3} \nabla_{y} w_{\xi} \cdot \nabla_{y} \phi d y=\int_{Y} \Lambda h e_{1} \cdot \nabla_{y} \phi-h^{3} \xi \cdot \nabla_{y} \phi d y, \quad \forall \phi \in W_{p e r}
$$


By linearity it follows that $w_{\xi}=v_{\xi}+v_{0}$, where $v_{\xi}$ and $v_{0}$ solve the local problems

$$
\begin{gathered}
\int_{Y} h^{3}\left(\xi+\nabla_{y} v_{\xi}\right) \cdot \nabla_{y} \phi d y=0, \quad \forall \phi \in W_{p e r} \\
\int_{Y}\left(\Lambda h e_{1}-h^{3} \nabla_{y} v_{0}\right) \cdot \nabla_{y} \phi d y=0, \quad \forall \phi \in W_{p e r} .
\end{gathered}
$$

Hence

$$
\begin{gathered}
f_{0}(x, \xi)=\int_{Y} \frac{1}{2} h^{3}\left(\xi+\nabla_{y} v_{\xi}+\nabla_{y} v_{0}\right) \cdot\left(\xi+\nabla_{y} v_{\xi}+\nabla_{y} v_{0}\right)-\Lambda h e_{1} \cdot\left(\xi+\nabla_{y} v_{\xi}+\nabla_{y} v_{0}\right) d y \\
=\frac{1}{2} \int_{Y} h^{3}\left(\xi+\nabla_{y} v_{\xi}\right) \cdot\left(\xi+\nabla_{y} v_{\xi}+\nabla_{y} v_{0}\right) d y \\
+\int_{Y}\left[h^{3} \nabla_{y} v_{0}-\Lambda h e_{1}\right] \cdot\left(\xi+\nabla_{y} v_{\xi}+\nabla_{y} v_{0}\right) d y-\int_{Y} \frac{1}{2} h^{3} \nabla_{y} v_{0} \cdot\left(\xi+\nabla_{y} v_{\xi}+\nabla_{y} v_{0}\right) d y .
\end{gathered}
$$

Since $v_{\xi}$ and $v_{0}$ are the solutions of the local problems (5) and (6) we get

$$
f_{0}(x, \xi)=\frac{1}{2} \int_{Y} h^{3}\left(\xi+\nabla_{y} v_{\xi}\right) \cdot \xi d y+\int_{Y}\left(h^{3} \nabla_{y} v_{0}-\Lambda h e_{1}\right) \cdot \xi d y-\int_{Y} \frac{1}{2} h^{3} \nabla_{y} v_{0} \cdot\left(\xi+\nabla_{y} v_{\xi}+\nabla_{y} v_{0}\right) d y
$$

Let the matrix function $A_{0}(x)$ and the vector function $B_{0}(x)$ be defined via

$$
\begin{gathered}
A_{0}(x) \xi=\int_{Y} h^{3}(x, y)\left(\xi+\nabla_{y} v_{\xi}(x, y)\right) d y, \\
B_{0}(x)=\int_{Y} \Lambda h(x, y) e_{1}-h^{3}(x, y) \nabla_{y} v_{0}(x, y) d y .
\end{gathered}
$$

By using the fact that $v_{\xi}$ is the solution of the local problem (5) we may write (8) as

$$
f_{0}(x, \xi)=\frac{1}{2} A_{0} \xi \cdot \xi-B_{0} \cdot \xi-\int_{Y} \frac{1}{2} h^{3} \nabla_{y} v_{0} \cdot \nabla_{y} v_{0} d y .
$$

Alternatively, by using that $v_{0}$ is the solution of the local problem (6) we could write

$$
f_{0}(x, \xi)=\frac{1}{2} A_{0}(x) \xi \cdot \xi-B_{0}(x) \cdot \xi-\frac{1}{2} \int_{Y} \Lambda h(x, y) e_{1} \cdot \nabla_{y} v_{0}(x, y) d y
$$

Both of these give us suitable formulas to compute $f_{0}(x, \xi)$.

In summary, for small values of $\varepsilon$ (i.e. small wavelengths) the pressure distribution $p_{\varepsilon} \rightarrow p_{0}$ in a suitable sense. Hence, $p_{\varepsilon}$ can be approximated by $p_{0}$ which is the solution of the minimization problem

$$
\min _{p} \int_{\Omega}\left(\frac{1}{2} A_{0}(x) \nabla p \cdot \nabla p-B_{0}(x) \cdot \nabla p-\frac{1}{2} \int_{Y} \Lambda h(x, y) e_{1} \cdot \nabla_{y} v_{0}(x, y) d y\right) d x
$$

and the corresponding Euler equation

$$
\nabla \cdot\left(A_{0}(x) \nabla p_{0}\right)=\nabla \cdot B_{0}(x) \quad \text { on } \Omega
$$

For small values of $\varepsilon$ the computation of the deterministic pressure $\left(p_{\varepsilon}\right)$ becomes impossible. Still, as described above, homogenization leads to a problem that considers the effects of surface roughness, which is possible to 
handle. However, due to the rather complex form of $f_{0}(x, \xi)$ involving the solution of the two local problems (5) and (6), much computational time is required when simulating realistic surface roughness representations. This paper applies a new method based on the use of a lower bound $f_{0}^{-}$and an upper bound $f_{0}^{+}$on $f_{0}(x, \xi)$, i.e.

$$
f_{0}^{-}(x, \xi) \leq f_{0}(x, \xi) \leq f_{0}^{+}(x, \xi)
$$

This means that we have the following bounds on the homogenized "energy" $F_{0}\left(\nabla p_{0}\right)$ :

$$
F_{0}^{-}\left(\nabla p^{-}\right) \leq F_{0}\left(\nabla p_{0}\right) \leq F_{0}^{+}\left(\nabla p^{+}\right)
$$

where

$$
F_{0}^{-}\left(\nabla p^{-}\right)=\min _{p} \int_{\Omega} f_{0}^{-}(x, \nabla p) d x
$$

and

$$
F_{0}^{+}\left(\nabla p^{+}\right)=\min _{p} \int_{\Omega} f_{0}^{+}(x, \nabla p) d x .
$$

In this paper, two different types of bounds are discussed. It will be demonstrated in situations likely to be present in realistic applications that the bounds are sufficiently close to each other to accurately estimate the pressure distribution $p_{0}$.

\section{Bounds}

As mentioned before, the study of finding bounds in mathematics and composite engineering has a long history. To our knowledge, these ideas and results have never been previously applied in the field of tribology. In this section, we discuss some ideas and results concerning bounds that may be used to analyze (1) and (2).

\subsection{Bounds of arithmetic-harmonic mean type}

Let us start by defining some functions considered important in the following discussion. Indeed, let either $(i, j)=$ $(1,2)$ or $(i, j)=(2,1)$ and define the following functions associated with the function describing the film thickness $h(x, y)$ :

$$
\begin{gathered}
a_{i}^{+}(x)=\left(\int_{0}^{1}\left(\int_{0}^{1} h^{3} d y_{j}\right)^{-1} d y_{i}\right)^{-1} \\
a_{i}^{-}(x)=\int_{0}^{1}\left(\int_{0}^{1} \frac{1}{h^{3}} d y_{i}\right)^{-1} d y_{j} \\
b^{+}(x)=a_{1}^{+}(x) \int_{0}^{1} \frac{\int_{0}^{1} \Lambda h d y_{2}}{\int_{0}^{1} h^{3} d y_{2}} d y_{1}
\end{gathered}
$$




$$
\begin{gathered}
b^{-}(x)=\int_{0}^{1}\left[\left(\int_{0}^{1} \frac{1}{h^{3}} d y_{1}\right)^{-1} \int_{0}^{1} \frac{\Lambda}{h^{2}} d y_{1}\right] d y_{2} \\
c^{+}(x)=\frac{1}{2} a_{1}^{+}(x)\left(\int_{0}^{1} \frac{\int_{0}^{1} \Lambda h d y_{2}}{\int_{0}^{1} h^{3} d y_{2}} d y_{1}\right)^{2}-\frac{1}{2} \int_{0}^{1} \frac{\left(\int_{0}^{1} \Lambda h d y_{2}\right)^{2}}{\int_{0}^{1} h^{3} d y_{2}} d y_{1} \\
c^{-}(x)=\frac{1}{2} \int_{0}^{1}\left[\left(\int_{0}^{1} \frac{1}{h^{3}} d y_{1}\right)^{-1}\left(\int_{0}^{1} \frac{\Lambda}{h^{2}} d y_{1}\right)^{2}\right] d y_{2}-\int_{Y} \frac{\Lambda^{2}}{2 h} d y
\end{gathered}
$$

The mathematical oriented work in [19] demonstrated that these functions determine the bounds on $f_{0}$. Here, these bounds are referred to as bounds of an arithmetic-harmonic mean type. For the readers convenience the main ideas in proving these bounds are summarized below, see [19] for details. The summary will also be important in our following discussion concerning a type of bounds, which are less precise, but has a very simple form.

Let $V \subset W_{\text {per }}$. It is then clear that

$$
f_{0}(x, \xi) \leq \min _{w \in V} \int_{Y} \frac{1}{2} h^{3}(x, y)\left(\xi+\nabla_{y} w\right) \cdot\left(\xi+\nabla_{y} w\right)-\Lambda h(x, y) e_{1} \cdot\left(\xi+\nabla_{y} w\right) d y .
$$

Thus the right hand side defines an upper bound $f_{0}^{+}(x, \xi)$ on $f_{0}(x, \xi)$ for any subset $V$ of $W_{\text {per }}$. The problem consist of finding a suitable subset $V$. That is, $f_{0}$ and $f_{0}^{+}$shall be as close as possible and finding an explicit expression for $f_{0}^{+}$should also be possible. If $V$ is chosen as

$$
V=\left\{\phi \in W_{p e r}: \phi(y)=\phi_{1}\left(y_{1}\right)+\phi_{2}\left(y_{2}\right)\right\}
$$

then

$$
f_{0}^{+}(x, \xi)=\frac{1}{2} a_{1}^{+} \xi_{1}^{2}+\frac{1}{2} a_{2}^{+} \xi_{2}^{2}-b^{+} \xi_{1}+c^{+}(x) .
$$

As seen below, this upper bound is an optimal choice in a certain sense.

The problem of finding a lower bound $f_{0}^{-}$on $f_{0}$ is more delicate, where the key ingredient is to find a dual (complementary) variational principle. Let $V_{\text {sol }}$ be the set of vector valued functions with zero divergence and mean value zero. It can then be proven that the conjugate function $f_{0}^{*}$ of $f_{0}$ is

$$
f_{0}^{*}(x, \eta) \stackrel{\text { def }}{=} \max _{s \in \mathbb{R}^{2}}\left\{\eta \cdot \xi-f_{0}(x, \xi)\right\}=\min _{\sigma \in V_{s o l}} \int_{Y} f^{*}(x, \eta+\sigma) d y
$$

where $f^{*}$ is the conjugate function corresponding to the integrand in $(15)$, i.e.

$$
f^{*}(x, y, t) \stackrel{\text { def }}{=} \max _{s \in \mathbb{R}^{2}}\left\{t \cdot s-\frac{1}{2} h^{3}(x, y) s \cdot s+\Lambda h(x, y) s_{1}\right\}=\frac{1}{2} h^{-3} t \cdot t+\Lambda h^{-2} t_{1}+\frac{1}{2} \Lambda^{2} h^{-1} .
$$

By arguing likewise, as for the upper bound of $f_{0}$, we now get an upper bound $f_{0}^{*+}$ for $f_{0}^{*}$ by choosing $\sigma$ only from a subset $V^{*}$ of $V_{\text {sol }}$ in 17 . This upper bound can then be used to obtain a lower bound $f_{0}^{-}$of $f_{0}$. Indeed, since $f_{0}$ is continuous and convex we have that $f_{0}=f_{0}^{* *}$, i.e.

$$
f_{0}(x, \xi)=\sup _{\eta}\left\{\xi \cdot \eta-f_{0}^{*}(x, \eta)\right\} \geq \sup _{\eta}\left\{\xi \cdot \eta-f_{0}^{*+}(x, \eta)\right\} .
$$


If $V^{*}$ is chosen as

$$
V^{*}=\left\{\sigma \in V_{\text {sol }}: \sigma=\left(\sigma_{1}\left(y_{2}\right), \sigma_{2}\left(y_{1}\right)\right)\right\}
$$

then the lower bound generated by (18) is

$$
f_{0}^{-}(x, \xi)=\frac{1}{2} a_{1}^{-} \xi_{1}^{2}+\frac{1}{2} a_{2}^{-} \xi_{2}^{2}-b^{-\xi_{1}}+c^{-}(x) .
$$

This also constitutes an optimal choice, as with the upper bound.

These bounds imply that the pressure $p^{-}$corresponding to the lower bound and the pressure $p^{+}$corresponding to the upper bound is found by solving the Euler equations

$$
\nabla \cdot\left(A^{ \pm} \nabla p^{ \pm}\right)=\frac{\partial b^{ \pm}}{\partial x_{1}} \text { on } \Omega \subset \mathbb{R}^{2},
$$

where

$$
A^{ \pm}=\left(\begin{array}{cc}
a_{1}^{ \pm} & 0 \\
0 & a_{2}^{ \pm}
\end{array}\right)
$$

\subsection{Optimality of the arithmetic-harmonic mean type bounds}

The bounds of arithmetic-harmonic mean type are optimal, i.e. there exists surface roughness descriptions $h$ for which the bounds $f_{0}^{-}$and $f_{0}^{+}$coincide with $f_{0}$. To see this, let us consider purely transversal surface roughness, i.e. the function $h_{1}(y)$ depends only on $y_{1}$. In this case, (9)-(12) yield the Euler equation coefficients

$$
\begin{aligned}
& a_{1}^{ \pm}=\left(\int_{0}^{1} h(x, y)^{-3} d y_{1}\right)^{-1} \\
& a_{2}^{ \pm}=\int_{0}^{1} h(x, y)^{3} d y_{1} \\
& b^{ \pm}=\Lambda \frac{\int_{0}^{1} h(x, y)^{-2} d y_{1}}{\int_{0}^{1} h(x, y)^{-3} d y_{1}} .
\end{aligned}
$$

The bounds also coincide in the case of longitudinal roughness, i.e. $h_{1}(y)$ depends on $y_{2}$ only, yielding the following Euler equation coefficients

$$
\begin{aligned}
& a_{1}^{ \pm}=\int_{0}^{1} h(x, y)^{3} d y_{2} \\
& a_{2}^{ \pm}=\left(\int_{0}^{1} h(x, y)^{-3} d y_{2}\right)^{-1} \\
& b^{ \pm}=\Lambda \int_{0}^{1} h(x, y) d y_{2} .
\end{aligned}
$$

Hence, for transversal and longitudinal roughness we have that $p^{-}=p^{+}=p_{0}$. Note that the formulas (21) and (22) are equivalent with those obtained in [5] and [7]. 


\subsection{Bounds of Reuss-Voigt type}

As illustrated above an important ingredient in proving arithmetic-harmonic type bounds was the choice of the subset $V$ of $W_{p e r}$ and the subset $V^{*}$ of $V_{\text {sol }}$. Obviously, by reducing both of these subsets to include only the zerofunction another set of bounds are obtained. This idea of reducing $V$ and $V^{*}$ has been used before in composite engineering, i.e. the derivation of the Reuss-Voigt bounds. Therefore, the bounds obtained here for the Reynolds equation will be referred to as bounds of Reuss-Voight type. Bounds of Reuss-Voigt type are of course less precise than the arithmetic-harmonic mean type of bounds, but lead to simple expressions for the coefficients of the corresponding Euler equations. In fact, by using (15) together with $V=\{0\}$ we get the upper bound of Reuss-Voigt type

$$
f_{0}(x, \xi) \leq \frac{1}{2} \alpha^{+}(x) \xi \cdot \xi-\beta^{+}(x) \xi_{1}
$$

where

$$
\begin{aligned}
& \alpha^{+}(x)=\int_{Y} h(x, y)^{3} d y, \\
& \beta^{+}(x)=\Lambda \int_{Y} h(x, y) d y .
\end{aligned}
$$

By using (17) and (18) with the choice $V^{*}=\{(0,0)\}$, the corresponding lower bound of Reuss-Voigt type is obtained

$$
f_{0}(x, \xi) \geq \frac{1}{2} \alpha^{-}(x) \xi \cdot \xi-\beta^{-}(x) \xi_{1}+\gamma^{-}(x),
$$

where

$$
\begin{aligned}
& \alpha^{-}(x)=\left(\int_{Y} h(x, y)^{-3} d y\right)^{-1}, \\
& \beta^{-}(x)=\Lambda \frac{\int_{Y} h(x, y)^{-2} d y}{\int_{Y} h(x, y)^{-3} d y}, \\
& \gamma^{-}(x)=\frac{\Lambda^{2}\left(\int_{Y} h(x, y)^{-2} d y\right)^{2}}{2 \int_{Y} h(x, y)^{-3} d y}-\frac{\Lambda^{2}}{2} \int_{Y} h(x, y)^{-1} d y .
\end{aligned}
$$

The pressure $p_{R V}^{-}$corresponding to the lower bound and the pressure $p_{R V}^{+}$corresponding to the upper bound may thus be obtained by solving the following Euler equations

$$
\nabla \cdot\left(\alpha^{ \pm}(x) \nabla p_{R V}^{ \pm}\right)=\frac{\partial \beta^{ \pm}(x)}{\partial x_{1}} \text { on } \Omega \subset \mathbb{R}^{2} .
$$

As mentioned before, the bounds of Reuss-Voigt type are by construction less precise than the bounds of the arithmetic-harmonic mean type. However, there are situations when the different types of bounds coincide. To see this, assume that the arithmetic mean

$$
\int_{0}^{1} h(x, y)^{3} d y_{j}
$$


is the same for all $y_{i}$, where $(i, j)=(1,2)$ or $(i, j)=(2,1)$. It is then easily seen that, e.g. $a_{1}^{+}(x)=a_{2}^{+}(x)=$ $\alpha^{+}(x)$. An example is given in the numerical experiments where surface roughness descriptions yield (28), being approximately the same for all $y_{i}$, see Fig. 9(left).

\section{Discretization}

To perform a numerical analysis, of the homogenization procedure previously described, a numerical method suitable for solving the different types of problems above was implemented in [[16]. Observe that the discretization technique presented considers time-dependent problems, which means that $q$ should be chosen as zero for the present numerical analysis. Note that the deterministic-, homogenized- and bounds- problems are of Dirichlet type, whereas the local problems are periodic.

For this purpose a partial differential equation of the type

$$
\frac{\partial}{\partial t}(q \Psi)=\nabla \cdot(\mathscr{A} \nabla \Psi)+\nabla \cdot(\Psi \mathscr{B})+f
$$

where

$$
\mathscr{A}(x, t)=\left(\begin{array}{ll}
a(x, t) & b(x, t) \\
c(x, t) & d(x, t)
\end{array}\right), \mathscr{B}(x, t)=\left(\begin{array}{l}
\lambda(x, t) \\
\gamma(x, t)
\end{array}\right),
$$

and

$$
q=q(x, t), \quad f=f(x, t),
$$

was considered.

The equation (29) is discretized using a second order central difference scheme in space. For example, this means that

$$
\begin{gathered}
\frac{\partial \Psi\left(x_{1}, x_{2}\right)}{\partial x_{1}} \approx \frac{1}{2 \Delta x_{1}}\left[\Psi_{i, j+1}-\Psi_{i, j-1}\right], \\
\frac{\partial}{\partial x_{1}}\left(a\left(x_{1}, x_{2}\right) \frac{\partial \Psi\left(x_{1}, x_{2}\right)}{\partial x_{1}}\right) \approx \frac{1}{\Delta x_{1}}\left[\left.a_{i, j+1 / 2} \frac{\partial \Psi}{\partial x_{1}}\right|_{i, j+1 / 2}-\left.a_{i, j-1 / 2} \frac{\partial \Psi}{\partial x_{1}}\right|_{i, j-1 / 2}\right] \approx \\
\frac{1}{\left(\Delta x_{1}\right)^{2}}\left[a_{i, j-1 / 2} \Psi_{i, j-1}-\left(a_{i, j-1 / 2}+a_{i, j+1 / 2}\right) \Psi_{i, j}+a_{i, j+1 / 2} \Psi_{i, j+1}\right] \approx
\end{gathered}
$$

and

$$
\begin{gathered}
\frac{\partial}{\partial x_{1}}\left(a\left(x_{1}, x_{2}\right) \frac{\partial \Psi\left(x_{1}, x_{2}\right)}{\partial x_{2}}\right) \approx \frac{1}{2 \Delta x_{1}}\left[\left.a_{i, j+1} \frac{\partial \Psi}{\partial x_{2}}\right|_{i, j+1}-\left.a_{i, j-1} \frac{\partial \Psi}{\partial x_{2}}\right|_{i, j-1}\right] \approx \\
\frac{1}{4 \Delta x_{1} \Delta x_{2}}\left[a_{i, j+1}\left(\Psi_{i+1, j+1}-\Psi_{i-1, j+1}\right)-a_{i, j-1}\left(\Psi_{i+1, j-1}-\Psi_{i-1, j-1}\right)\right] .
\end{gathered}
$$

Combined with an implicit second order upwind discretization in time, the discrete representation of Eq. (29) yields

$$
\frac{1}{2 \Delta t}\left(3 q_{i, j}^{k} \Psi_{i, j}^{k}-4 q_{i, j}^{k-1} \Psi_{i, j}^{k-1}+q_{i, j}^{k-2} \Psi_{i, j}^{k-2}\right)=
$$




$$
\begin{gathered}
\frac{1}{\left(\Delta x_{1}\right)^{2}}\left[a_{i, j-1 / 2}^{k} \Psi_{i, j-1}^{k}-\left(a_{i, j-1 / 2}^{k}+a_{i, j+1 / 2}^{k}\right) \Psi_{i, j}^{k}+a_{i, j+1 / 2}^{k} \Psi_{i, j+1}^{k}\right]+ \\
\frac{1}{4 \Delta x_{1} \Delta x_{2}}\left[b_{i, j+1}^{k}\left(\Psi_{i+1, j+1}^{k}-\Psi_{i-1, j+1}^{k}\right)-b_{i, j-1}^{k}\left(\Psi_{i+1, j-1}^{k}-\Psi_{i-1, j-1}^{k}\right)\right]+ \\
\frac{1}{4 \Delta x_{2} \Delta x_{1}}\left[c_{i+1, j}^{k}\left(\Psi_{i+1, j+1}^{k}-\Psi_{i+1, j-1}^{k}\right)-c_{i-1, j}^{k}\left(\Psi_{i-1, j+1}^{k}-\Psi_{i-1, j-1}^{k}\right)\right]+ \\
\frac{1}{\left(\Delta x_{2}\right)^{2}}\left[d_{i-1 / 2, j}^{k} \Psi_{i-1, j}^{k}-\left(d_{i-1 / 2, j}^{k}+d_{i+1 / 2, j}^{k}\right) \Psi_{i, j}^{k}+d_{i+1 / 2, j}^{k} \Psi_{i+1, j}^{k}\right]+ \\
\frac{1}{2 \Delta x_{1}}\left[\lambda_{i, j+1}^{k} \Psi_{i, j+1}^{k}-\lambda_{i, j-1}^{k} \Psi_{i, j-1}^{k}\right]+\frac{1}{2 \Delta x_{2}}\left[\gamma_{i+1, j}^{k} \Psi_{i+1, j}^{k}-\gamma_{i-1, j}^{k} \Psi_{i-1, j}^{k}\right]+f_{i, j}^{k}
\end{gathered}
$$

where $a_{i, j \pm 1 / 2}=\left(a_{i, j}+a_{i, j \pm 1}\right) / 2$ and $a_{i \pm 1 / 2, j}=\left(a_{i, j}+a_{i \pm 1, j}\right) / 2$.

To compute the coefficients determining the Euler equations in the different bounds problems, numerical integration based on Simpson's rule was adopted. This means that, e.g.

$$
\int_{\Omega} f(x) d x \approx \frac{\Delta x}{3} \sum_{l=2}^{N-1} f_{l-1}+4 f_{l}+f_{l+1}
$$

where $f_{i}=f\left(x_{i}\right)$ and $N$ is the number of discrete grid nodes $x_{i}$.

\section{Numerical results and discussion}

In this section, it is demonstrated that the bounds technique may be used to efficiently analyze the effects of surface roughness. Four model problems are considered, two where explicit mathematical expressions are used to model the surface roughness and two where the surface roughness originates from measurements. In all cases the solution domain $\Omega$ is the set of $x \in \mathbb{R}^{2}$ such that $0 \leq x_{1} \leq L_{1}$ and $-L_{2} / 2 \leq x_{2} \leq L_{2} / 2$. The global part of the film thickness described by

$$
h_{0}(x)=h_{\min }\left(-k \frac{x_{1}}{L_{1}}+1+k\right)
$$

were also preserved together with some specific parameters found in Table 11 We will also use a parameter $c$ to

Table 1: Problem specific parameters

\begin{tabular}{lll}
\hline Parameter & Value & Unit \\
\hline$h_{\min }$ & $4 \cdot 10^{-6}$ & $\mathrm{~m}$ \\
$k$ & $1 / 4$ & \\
$L_{1}=L_{2}$ & $1 \cdot 10^{-1}$ & $\mathrm{~m}$ \\
$U$ & 1 & $\mathrm{~m} \mathrm{~s}^{-1}$ \\
$\mu$ & $1.4 \cdot 10^{-1}$ & $\mathrm{~Pa} \mathrm{~s}$ \\
\hline
\end{tabular}

describe the amplitude of the surface roughness and if nothing else is specifically mentioned $c=1 / 4$. 
Case 1: A pure transversal sinusoidal roughness description is assumed. More precisely,

$$
h_{1}(y)=h_{\min } \frac{c}{2}\left(1-\sin \left(2 \pi y_{1}\right)\right) .
$$

In Fig. 2 the homogenized pressure is visualized. As described above the deterministic pressure $p_{\varepsilon}$ converges to the

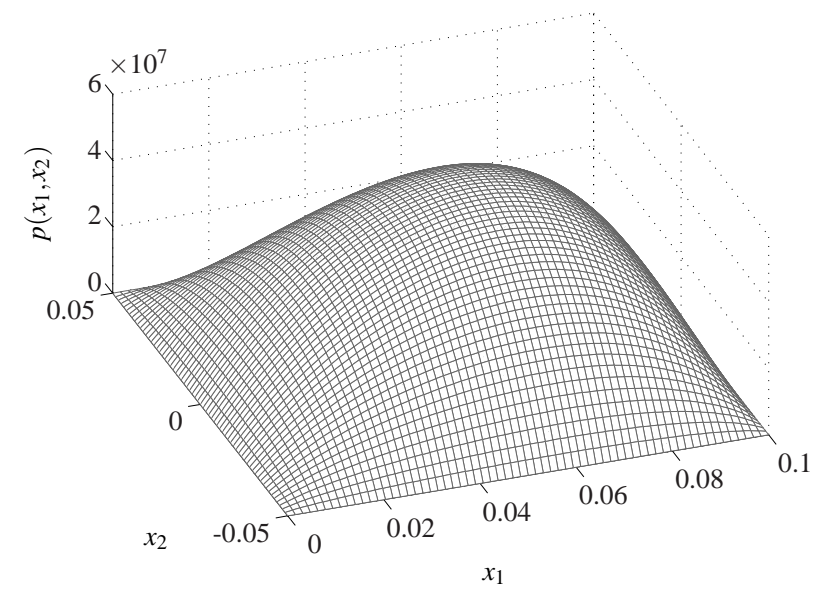

Figure 2: The homogenized pressure solution in the case of pure transversal sinusoidal roughness

homogenized pressure $p_{0}$ as $\varepsilon$ tends to 0 . This fact is illustrated in Fig. 3, where the deterministic pressure solutions (for three different values of $\varepsilon$ ) and the homogenized pressure are plotted along the centerline. As previously noted,

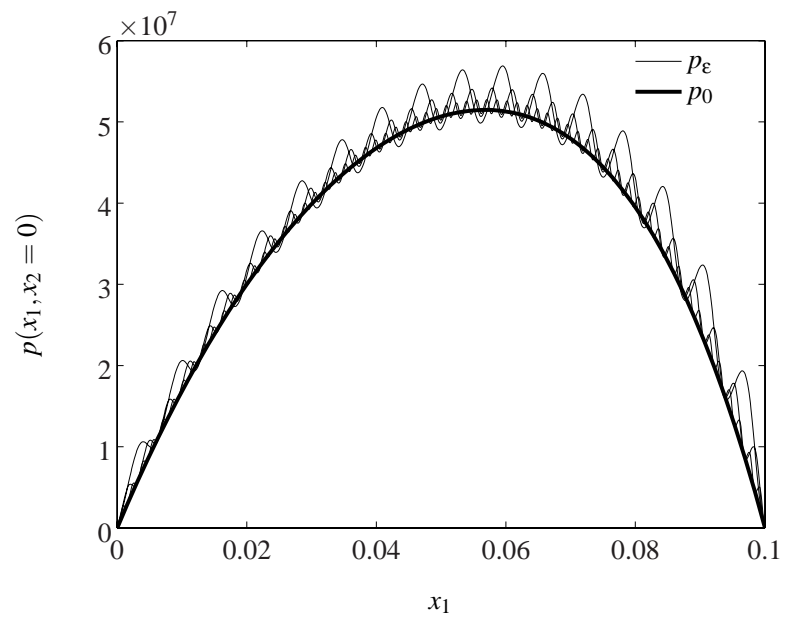

Figure 3: The deterministic- $\left(p_{\varepsilon}\right)$ for three choices of $\varepsilon$, i.e. $2^{-4}, 2^{-5}, 2^{-6}$, and the homogenized- $\left(p_{0}\right)$ pressure solution(s) at the centerline in the case of pure transversal sinusoidal roughness

the arithmetic-mean type of bounds coincide in the case of a transversal roughness description, i.e. $p_{0}=p^{+}=p^{-}$. In Fig. 4 the homogenized pressure solution $p_{0}$ and the Reuss-Voigt bounds pressure solutions $p_{R V}^{+}$and $p_{R V}^{-}$are plotted. From the figure it is clear that the Reuss-Voigt type bounds, for the input parameters chosen, serves as a suitable estimate of the homogenized pressure. In the figure, the influence of surface roughness on the pressure 
distribution is illustrated by the solutions $p_{\min }$ and $p_{\max }$. These are obtained assuming smooth surfaces described by $h_{0}(x)+\min _{y} h_{1}(y)$ and $h_{0}(x)+\max _{y} h_{1}(y)$. The difference between the Reuss-Voigt estimates, measured in terms

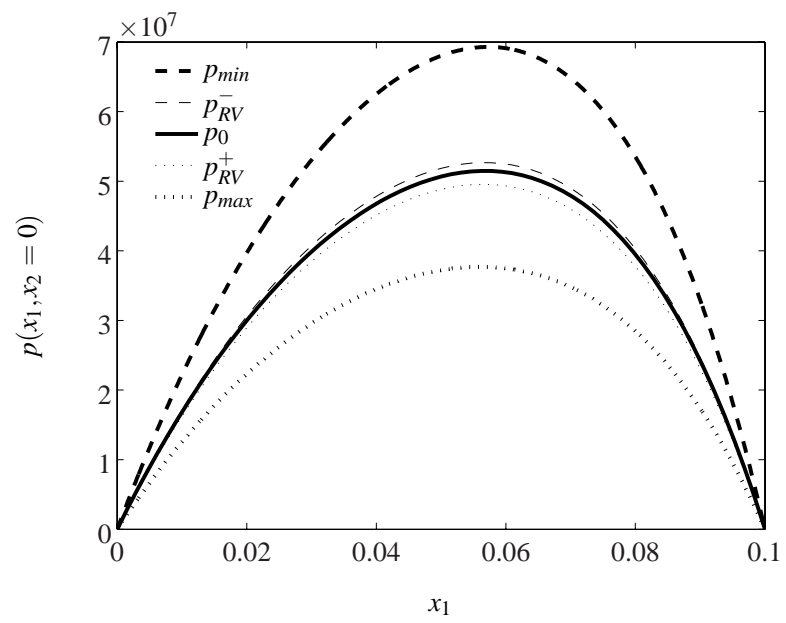

Figure 4: The Reuss-Voigt estimates $\left(p_{R V}^{-}\right)$and $\left(p_{R V}^{+}\right)$, the homogenized- $\left(p_{0}\right)$ pressure solution(s) and the solutions $p_{\max }$ and $p_{\min }$ at the centerline in the case of pure transversal sinusoidal roughness

of load carrying capacity, is

$$
\delta=\frac{\int_{\Omega}\left(p_{R V}^{-}-p_{R V}^{+}\right) d x}{\int_{\Omega} p_{0} d x}=6 \cdot 10^{-2} .
$$

In other words, the Reuss-Voigt estimates predict the homogenized solution (and $p^{-}$and $p^{+}$, which are identical) within an accuracy of approximately $6 \%$. In comparison, the difference

$$
\frac{\int_{\Omega}\left(p_{\max }-p_{\min }\right) d x}{\int_{\Omega} p_{0} d x}=60 \cdot 10^{-2},
$$

emphasizes the importance of rough surface modeling.

Case 2: Next a mathematical description of surface roughness according to

$$
h_{1}(y)=h_{\min } \frac{c}{2}\left(1-\cos \left(2 \pi y_{1}\right) \cos \left(2 \pi y_{2}\right)\right) \text {, }
$$

was investigated, again with the slider defined by (30). The deterministic $\left(p_{\varepsilon}\right)$ pressure solution for the choice of $\varepsilon=2^{-4}$ is visualized in Fig. 5. In this case, the differences between the Reuss-Voigt solutions $\left(p_{R V}^{-}\right.$and $\left.p_{R V}^{+}\right)$and the arithmetic-harmonic solutions $\left(p^{-}\right.$and $\left.p^{+}\right)$are small according to

$$
\frac{\int_{\Omega}\left(p^{-}-p^{+}\right) d x}{\int_{\Omega} p_{0} d x}=3.03 \cdot 10^{-2}
$$

and

$$
\frac{\int_{\Omega}\left(p_{R V}^{-}-p_{R V}^{+}\right) d x}{\int_{\Omega} p_{0} d x}=3.06 \cdot 10^{-2} .
$$

Hence, both types of bounds may be used to obtain suitable approximations of the homogenized problem. This is also graphically illustrated by Fig. 6, in which the bounds' solutions at the centerline are visualized. 


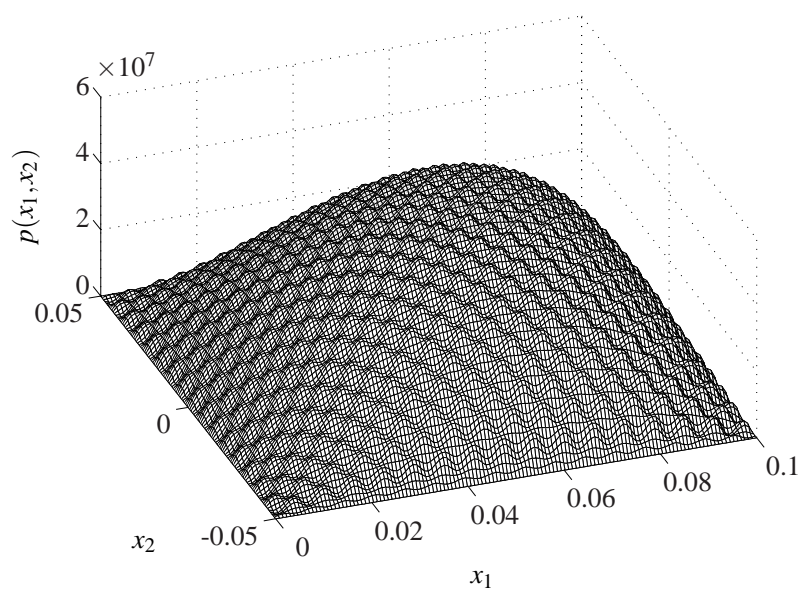

Figure 5: The deterministic $\left(p_{\varepsilon}\right)$ pressure solution for the choice of $\varepsilon=2^{-4}$ with surface roughness described by 34

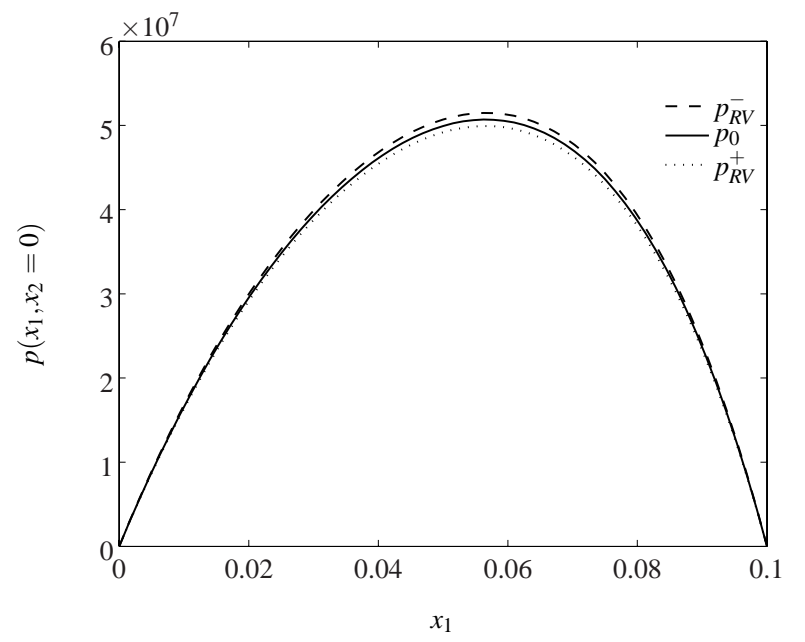

Figure 6: The Reuss-Voigt $\left(p_{R V}^{-}\right.$and $\left.p_{R V}^{+}\right)$and the arithmetic-harmonic $\left(p^{-}\right.$and $\left.p^{+}\right)$pressure solutions at the centerline, with the surface roughness described by (34) 
The last two examples are based on the surface roughness descriptions shown in Figures 7 (left) and 9 (left). These are actually taken from measurements and has been chosen for the purpose of having realistic representations of striated- (transversal, longitudinal or with a specific lay) and isotropic- roughness.

In the numerical analysis these topographies has been used to represent the $Y$-cell, which describes the periodicity of the function $h_{1}$. An interpolation method may be used to reduce the number of local problems to be solved in order to retrieve the coefficients of the homogenized equation. However, the number of degrees of freedoms introduced by a realistic surface description is large, which means that it may become difficult or even impossible to solve a single local problem with sufficiently high numerical accuracy.

Using the ideas presented here, directly lowers the computational burden since the coefficients of the Euler equations for the different types of bounds are obtained by numerical integration (and no PDE defined on the local scale has to be solved).

The surface heights of both these surfaces are normalized $([0,1])$ and then scaled by $h_{\min }$ and $c$ to yield the surface roughness representations $h_{1}$ used in the numerical examples.

Case 3: Here the surface roughness description $h_{1}$, used for the numerical analysis, is given by Fig. 7(left) and the bounds solutions (both arithmetic-harmonic and Reuss-Voigt types) are visualized in Fig. 7 (right). The differences
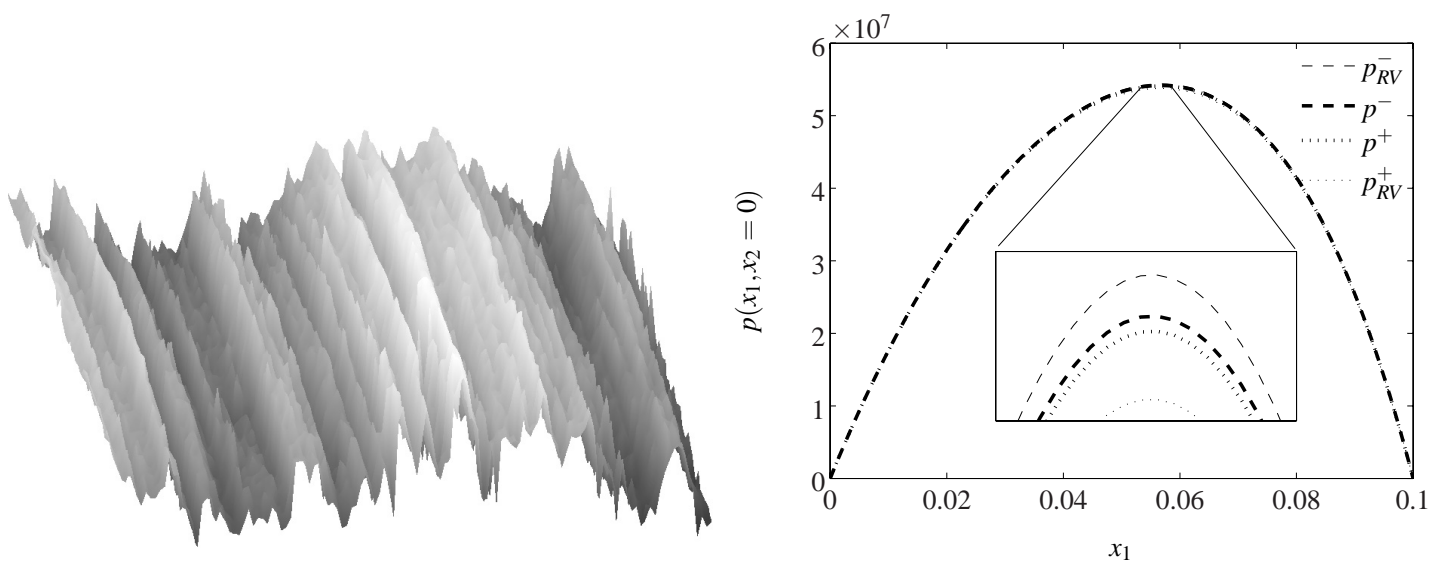

Figure 7: A striated surface roughness representation used as $h_{1}$ for the numerical analysis (left). The Reuss-Voigt $\left(p_{R V}^{-}\right.$and $\left.p_{R V}^{+}\right)$and the arithmetic-harmonic $\left(p^{-}\right.$and $\left.p^{+}\right)$pressure solutions at the centerline (right).

are small between the different solutions and an enlargement is added to the figure giving some insight. Of note,

$$
2 \frac{\int_{\Omega}\left(p^{-}-p^{+}\right) d x}{\int_{\Omega}\left(p^{-}+p^{+}\right) d x} \approx 1.5 \cdot 10^{-4}
$$

and

$$
2 \frac{\int_{\Omega}\left(p_{R V}^{-}-p_{R V}^{+}\right) d x}{\int_{\Omega}\left(p_{R V}^{-}+p_{R V}^{+}\right) d x} \approx 1.2 \cdot 10^{-2} .
$$

This agrees with the previous discussion about the optimality of the bounds in the case of pure transversal- or longitudinal roughness since the surface found in Fig. 7(left) varies 10 times more in amplitude in one direction 
than in the other. However, it seems that even the crude estimates quite accurately predict the homogenized solution (within an accuracy of $1.2 \%$ ).

Next an investigation showing the effects on the surface roughness amplitude relatively to the minimum global film thickness was performed. In this investigation, the arithmetic-harmonic bounds solutions are compared to the solution that appears to be the first choice of an engineer, i.e. the smooth solution $\bar{p}$ obtained by taking the local average of the gap. More precisely, $\bar{p}$ solves the following equation

$$
\nabla \cdot\left(\bar{h}^{3}(x) \nabla \bar{p}(x)\right)=\Lambda \frac{\partial \bar{h}(x)}{\partial x_{1}} \quad \text { on } \Omega \subset \mathbb{R}^{2},
$$

where

$$
\bar{h}=\int_{Y} h(x, y) d y .
$$

The results, in terms of pressure solution at the centerline, are shown in Fig. 8 .
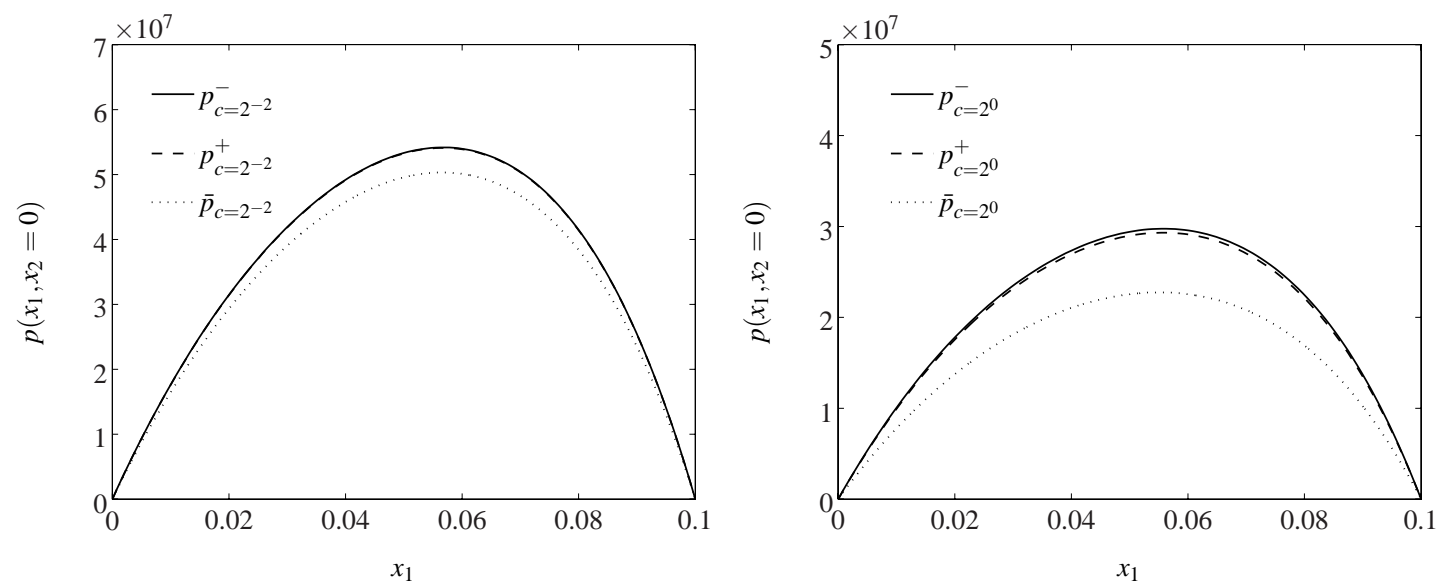

Figure 8: The arithmetic-harmonic solutions $\left(p_{c}^{ \pm}\right)$and the corresponding smooth average solution $\left(\bar{p}_{c}\right)$ using the striated surface roughness description in the left of Fig. 7 for $c=1 / 4$ (left), $c=1$ (right).

Case 4: The roughness visualized in Fig. 9 (left) is used in this example. Here, it becomes quite clear that the differences between the Reuss-Voigt- and the arithmetic-harmonic- solutions are small Fig. 9 (right). Again, the homogenized solution is predicted very precisely with an accuracy of approximately $0.8 \%$. Similarly to the investigation performed in Case 3, showing the effects on the surface roughness amplitude relatively to the minimum global film thickness was also conducted in this case of a realistic representation of an isotropic surface roughness. Again, the arithmetic-harmonic bounds solutions are compared to the smooth solution $\bar{p}$, at the centerline, with results shown in Fig. 10

To generalize the result, the problem was transformed into a non-dimensional form by the set of dimensionless variables given by

$$
X_{i}=x_{i} / L_{1}, \quad H=h / h_{\text {min }}, \quad P=p /\left(\frac{\Lambda L_{1}}{h_{\text {min }}^{2}}\right) .
$$



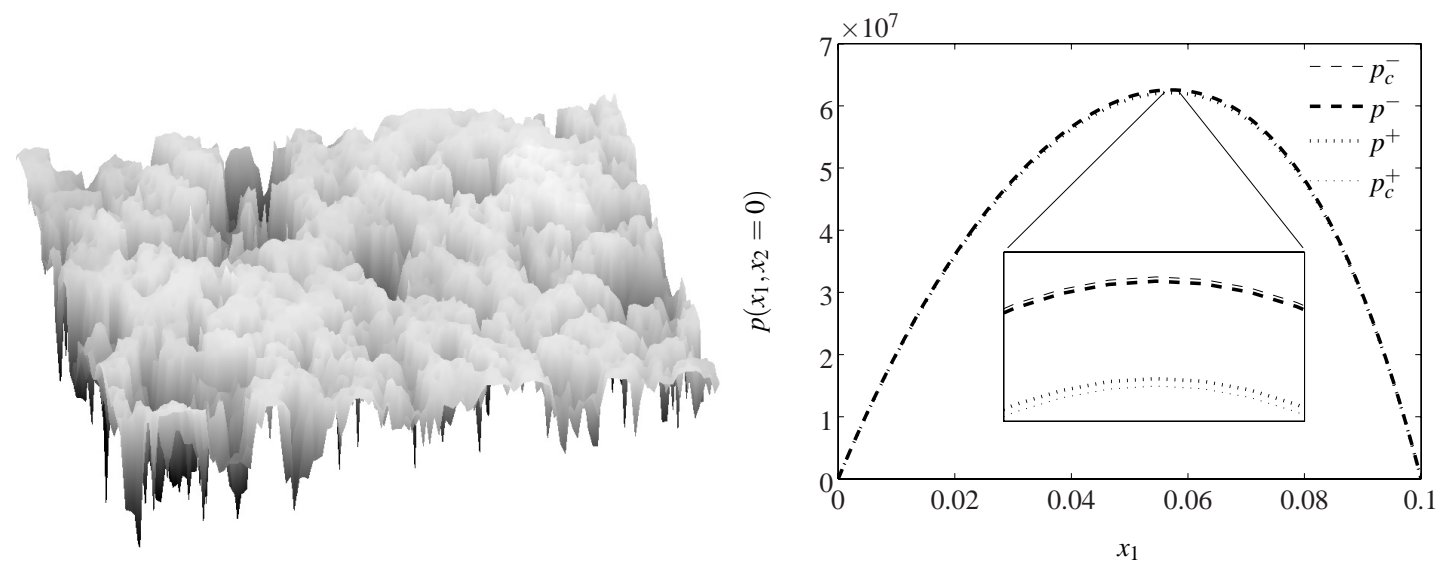

Figure 9: An isotropic surface roughness representation used as $h_{1}$ for the numerical analysis (left). The ReussVoigt $\left(p_{R V}^{-}\right.$and $\left.p_{R V}^{+}\right)$and the arithmetic-harmonic $\left(p^{-}\right.$and $\left.p^{+}\right)$pressure solutions at the centerline (right).
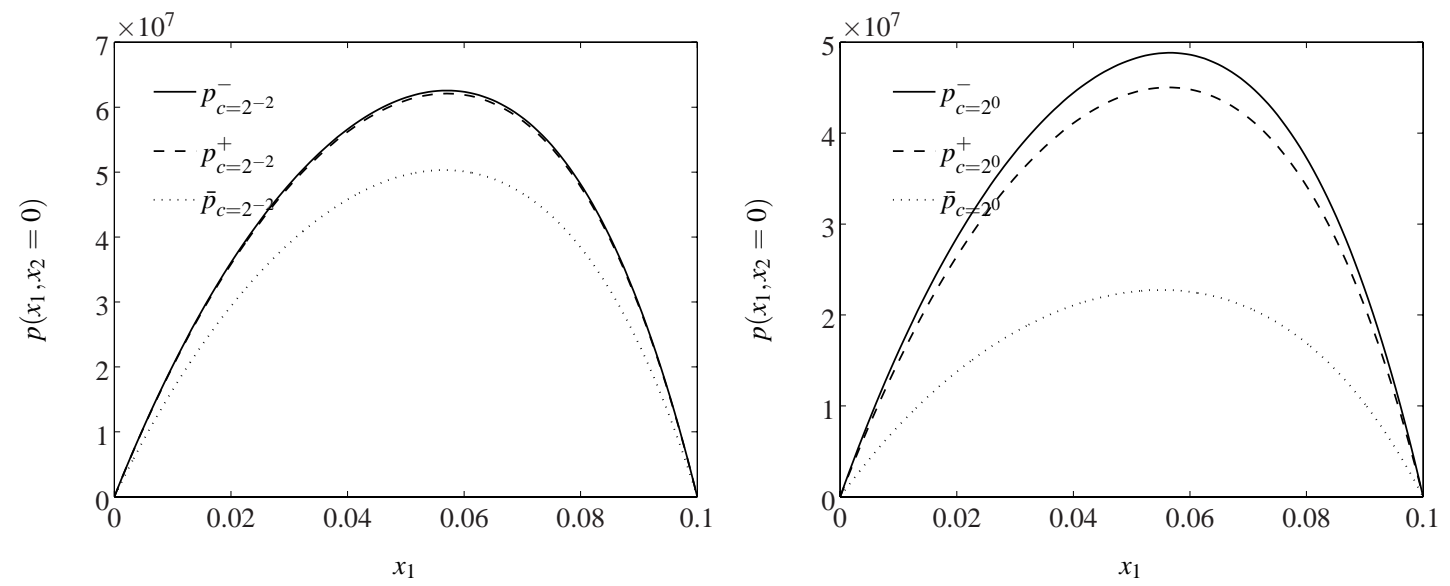

Figure 10: The arithmetic-harmonic solutions $\left(p_{c}^{ \pm}\right)$and the corresponding smooth average solution $\left(\bar{p}_{c}\right)$ using the isotropic surface roughness description in the left of Fig. 9 for $c=1 / 4$ (left), $c=1$ (right). 
This yields a two parameter problem, i.e. $k$ and $c$ becomes the only parameters determining the pressure solution. Using the non-dimensional description, an investigation of change in accuracy $(\delta)$ with roughness amplitude $(c)$ was performed. It was found that the variation of $\delta$ with $c$ is approximately a relation on the form

$$
\delta=C c^{\gamma} .
$$

With a correlation of $82-94 \%$ for $c \leq 1$, the exponent $\gamma$ was found to be approximately equal to $1.80-1.87$. This expression is valid for any choice of the parameters $\Lambda, L_{1}$ and $h_{\min }$, meaning that the accuracy of the estimate is not affected by these three parameters. Further more, it was verified that (42) holds for all four surface roughness descriptions used here, posed as

$$
h_{1}(y)=h_{\min } c \phi(y) .
$$

where $0 \leq \phi(y) \leq 1$ is a normalized representation of the actual surface roughness. For example, let us recall the results from the last example where the accuracy of the Reuss-Voigt estimate was found to be $0.8 \%$. In this case $c=1 / 4$. However, according to (42) the accuracy is $<10 \%$ if the roughness amplitude equals the global minimum film thickness.

In summary, given a set of operational conditions it is possible to determine the accuracy of the estimate by performing a single simulation of the application specific surface roughness.

\section{Concluding remarks}

We have studied the influence of surface roughness on hydrodynamic performance. The main tool has been to use bounds on the averaged properties obtained by homogenization. To our knowledge, this is the first time the usefulness of this technique has been demonstrated in the theory of lubrication. Because of the positive outcome, we believe that the application of bounds in tribology deserves further studies. Especially, we want to draw the attention to that in this work the analysis has been restricted to include problems where the moving surface is assumed to be smooth. Hence, an important development would be to consider the situation where both surfaces are rough, leading to time dependent problems. Another development would be to study variational inequalities to

be able to consider cavitation. It should be noted that time dependent Reynolds type problems recently have been analyzed by homogenization, though the ideas of bounds have never been used. 


\section{References}

[1] N. Patir and H. S. Cheng. An average flow model for determining effects of three-dimensional roughness on partial hydrodynamic lubrication. Proc. 5th Leeds-Lyon Symp. Tribol., Lyon, pages 15-21, 1978.

[2] V.V. Jikov, S.M. Kozlov, and O.A. Oleinik. Homogenization of Differential Operators and Integral Functionals. Springer-Verlag, Berlin-Heidelberg-New York, 1994.

[3] A. Almqvist and J. Dasht. The homogenization process of the reynolds equation describing compressible flow. Tribology International, 39:994-1002, 2006.

[4] H. G. Elrod. Thin-film lubrication theory for newtonian fluids with surfaces possessing striated roughness or grooving. ASME J. Lubr. Technol., 95:484-489, 1973.

[5] G. Bayada and J. B. Faure. A double scale analysis approach of the reynolds roughness comments and application to the journal bearing. Journal of Tribology, 111:323-330, 1989.

[6] M. Kane and B. Bou-Said. Comparison of homogenization and direct techniques for the treatment of roughness in incompressible lubrication. Journal of Tribology, 126(4):733-737, oct 2004.

[7] P. Wall. Homogenization of reynolds equation by two-scale convergence. Research Report, No. 2, ISSN 1400-4003, Department of Mathematics, Luleå University of Technology, 2005.

[8] G. Bayada, S. Ciuperca, and M. Jai. Homogenization of variational equations and inequalities with small oscillating parameters. application to the study of thin film unstationary lubrication flow. C. R. Acad. Sci. paris, t. 328, Série II b:819-824, 2000.

[9] G. C. Buscaglia and M. Jai. Sensitivity analysis and taylor expansions in numerical homogenization problems. Numerische Mathematik, 85:49-75, 2000.

[10] G. C. Buscaglia and M. Jai. A new numerical scheme for non uniform homogenized problems: Application to the non linear reynolds compressible equation. Mathematical Problems in Engineering, 7:355-378, 2001.

[11] G. C. Buscaglia, I. Ciuperca, and M. Jai. Homogenization of the transient reynolds equation. Asymptotic Analysis, 32(2):131-52, Nov 2002.

[12] G. C. Buscaglia and M. Jai. Homogenization of the generalized reynolds equation for ultra-thin gas films and its resolution by fem. Journal of Tribology, 126(3):547-552, jul 2004.

[13] M. Jai. Homogenization and two-scale convergence of the compressible reynolds lubrication equation modelling the flying characteristics of a rough magnetic head over a rough rigid-disk surface. ASME Journal of Tribology, 124:327-335, 2002. 
[14] M. Jai and B. Bou-Said. A comparison of homogenization and averaging techniques for the treatment of roughness in slip-flow-modified reynolds equation. Transactions of the ASME. Journal of Tribology, 124(2):327-335, April 2002.

[15] M. Kane and B. Bou-Said. A study of roughness and non-newtonian effects in lubricated contacts. ASME Journal of Tribology, 127:575-581, 2005.

[16] A. Almqvist, R. Larsson, and P. Wall. The homogenization process of the time dependent reynolds equation describing compressible liquid flow. Research Report, No. 4, ISSN 1400-4003, Department of Mathematics, Luleå University of Technology, Submitted for publication in Tribology International, 2006.

[17] G. W. Milton. The Theory of Composites. Cambridge University Press, Cambridge, United Kingdom, 2002.

[18] S. Torquato. Random Heterogeneous Materials. Springer-Verlag New York, Inc., 2002.

[19] D. Lukkassen, A. Meidell, and P. Wall. Bounds on the effective behavior of a homogenized reynold-type equation. Research Report, No. 3, ISSN 1400-4003, Department of Mathematics, Luleå University of Technology, Accepted for publication in Journal of Function Spaces and Applications, 2006. 